D.T. Wickramasinghe, L. Ferrario, and G.V. Bicknell, eds.

\title{
Cygnus X-1: A Case for a Magnetic Accretion Disk?
}

\author{
Michael A. Nowak \\ JILA, Campus Box 440, Boulder, CO 80309-0440 \\ B. A. Vaughan \\ Caltech, Pasadena, CA 91125 \\ J. Dove \\ also Dept. of APAS, University of Colorado, Boulder, CO 80309 \\ J. Wilms
}

\section{IAA University of Tübingen, Waldheuser Str. 64, Tübingen, Germany}

\begin{abstract}
With the advent of RXTE, which is capable of broad spectral coverage and fast timing, as well as other instruments which are increasingly being used in multi-wavelength campaigns (via both spacebased and ground-based observations), we must demand more of our theoretical models. No current model mimics all facets of a system as complex as an x-ray binary. However, a modern theory should qualitatively reproduce - or at the very least not fundamentally disagree with - all of Cygnus X-1's most basic average properties: energy spectrum (viewed within a broader framework of black hole candidate spectral behavior), power spectrum (PSD), and time delays and coherence between variability in different energy bands. Below we discuss each of these basic properties in turn, and we assess the health of one of the currently popular theories: Comptonization of photons from a cold disk. We find that the data pose substantial challenges for this theory, as well as all other currently discussed models.
\end{abstract}

\section{Energy Spectra}

Historically, energy spectra have been the primary focus of both observers and theorists. The low state spectrum of Cyg X-1 (see Fig. 1) for the most part is well-fit by a power-law with a photon index $\sim 1.7$. Additional facts to keep in mind are: the total luminosity of Cyg X-1 is likely $<0.1 L_{\mathrm{Edd}}$, and that most other black holes show a strong soft component and softer power-law tail above $>0.1 L_{E d d}$ (cf. Nowak 1995). The softer, high state of Cyg X-1 is of roughly comparable luminosity to the low state (Zhang 1996). The soft components that do exist in the low state are weak compared to the power-law.

The most popular models for this state are hot, optically thin models [with advection dominated disks (ADD) being one example, cf. Narayan et al. 1996], and Comptonization of seed photons from a cold disk (cf. Haardt \& Maraschi 


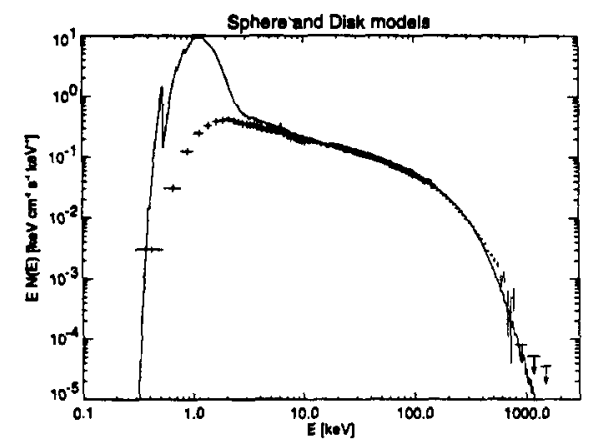

Figure 1. Best-fit corona model to unfolded, non-simultaneous Cyg X-1 spectrum. Data are from BBXRT, TTM, (simultaneous with) HEXE, and OSSE (Dove, Wilms, \& Begelman 1996).

1993; Dove, Wilms, \& Begelman 1996). ADD theories provide an explanation for the hard to soft transition at $\sim 0.1 L_{E d d}$, although they have yet to selfconsistently calculate the (weak) observed features that Comptonization models attribute to reflection. One theoretical motivation for the Comptonization models is the recent work (cf. Balbus, et al. 1996; Hawley et al. 1996) demonstrating the existence of a powerful magnetic field-driven shear instability that is strongest in low-density regions. Comptonization models assume that most of the disk energy is dissipated in a corona, with roughly half being reprocessed by the disk into soft photons (cf. Haardt \& Maraschi 1993).

Fitting the spectra of Cyg X-1 above $2 \mathrm{keV}$ typcally requires $\tau_{e s} \sim 0.2$ and $k T_{e} \sim 150 \mathrm{keV}$. With roughly half of the luminosity being reprocessed by the disk, and a large fraction escaping the optically thin corona, we expect a large soft flux at $\sim 200 \mathrm{eV}$. This is not observed (see Fig. 1 and Dove, Wilms, Begelman 1996, where more refined models will be presented). In addition to not offering an explanation for the low-high transition at $\sim 0.1 L_{E d d}$, this is a severe problem for the corona models. The models do, however, correctly account for the Fe-line and reflection features, with $\sim$ half of the soft $200 \mathrm{eV}$ excess being due to reflection (as opposed to intrinsic disk emission). ADD models, with a similar geometry and a strong source of hard photons, may also overproduce soft X-rays if they attempt to model the observed Fe-line with reflection off of the optically thick, geometrically thin outer disk.

\section{Phase/Time Lags Between Soft and Hard Variability}

Cyg X-1 shows strong X-ray variability (rms fluctuations $\sim 40 \%$ ), with timescales ranging from milliseconds to tens of seconds. This variability is usually measured by Fourier transforming the observed time series and then forming the power spectral density (PSD), i.e. the squared transform. One can also form the cross power density (CPD) by multiplying together the complex transforms from two time series. The Fourier frequency-dependent phase of the CPD is known as the phase delay, $\theta_{d}(f)$, from which one can form the time delay, $\tau_{d} \equiv \theta_{d} / 2 \pi f$. Cygnus X-1 shows a characteristic time lag of the hard photons behind the soft, 

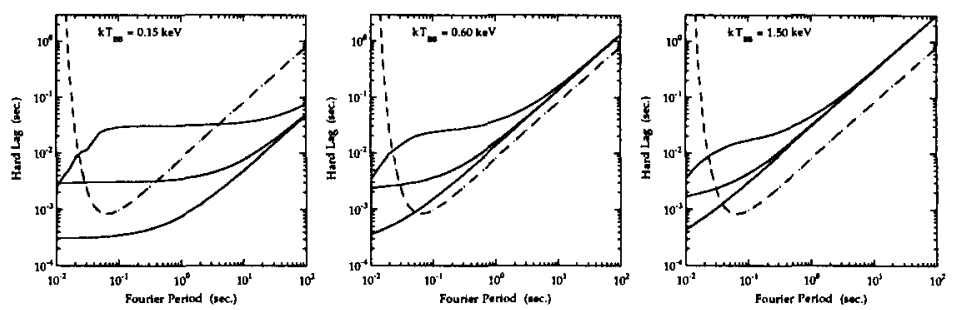

Figure 2. Effects of Compton coronae on intrinsic phase lags, as a function of input blackbody temperature and mean scattering path length $\left(\lambda_{e s}=10^{6}, 10^{7}\right.$, and $\left.10^{8} \mathrm{~cm}\right)$. From Nowak \& Vaughan (1996).

with $\tau_{d} \sim 2(f / 0.01 \mathrm{~Hz})^{-2 / 3} \mathrm{~s}$ (cf. Miyamoto et al. 1992). This corresponds to a nearly constant phase delay of $\sim 0.1 \mathrm{rad}$.

The only currently proposed ADD model explanation for this delay invokes thermal disturbances propagating from the outer disk edge toward the center (cf. Manmoto et al. 1996). This model requires, however, a very slow propagation speed $\sim 10^{-4} c$ in order to be in quantitative aggreement with observations (cf. Vaughan \& Nowak 1996). Corona models explain the delay as possibly arrising from the magnetic flares that are allegedly energizing the corona (cf. Nowak 1994). If the flare temperature increases throughout its evolution, hard photons naturally lag soft photons. However, diffusion of photon scattering times within the corona will lead to a "smearing out" of this intrinsic time lag, depending upon the spectrum of the seed photons, the corona temperature, optical depth, and mean free scattering path (cf. Fig.2; Miller 1995; Nowak \& Vaughan 1996). Current observations rule out large scattering paths $\left(\sim 10^{8} \mathrm{~cm}\right)$ with very soft $(\sim 150 \mathrm{eV})$ inputs. Upcoming RXTE observations (obtaining phase lags in 6-8 energy channels out to $\sim 250 \mathrm{~Hz}$ ) will provide more stringent constraints.

\section{Coherence Between Soft and Hard Variability}

The coherence function is essentially the normalized average amplitude of the CPD (cf. Vaughan \& Nowak 1996, Nowak \& Vaughan 1996). It is a measure of the fraction of the two time series that can be interpreted as time-independent and linear transforms of one another. Coherence must be less than or equal to unity, and be equal to unity only when there is a linear, time-indpendent transfer function relating one channel to the other. Coherence is less than unity under any of the following conditions: variability is due to thermal flares that are dominated by emission from the Wien tale; there are multiple, independent sources of variability (whether linear or not) with multiple, independent responses to the sources (whether linear of not); there is any non-linear transfer function from one channel to the other (cf. Vaughan \& Nowak 1996).

If coronae are truly formed via multiple magnetic flares, then we expect a strong loss of coherence, unless the the response to each of the flares is identical. Nowak (1994) showed that a kinematic model, with multiple flaring regions, reproduced both the PSD and phase lags seen in the very high state of GX3394 , as well as qualititatively agreed with the energetics of coronal formation. This model, and all others like it, produces a strong loss of coherence (Fig. 3). This 

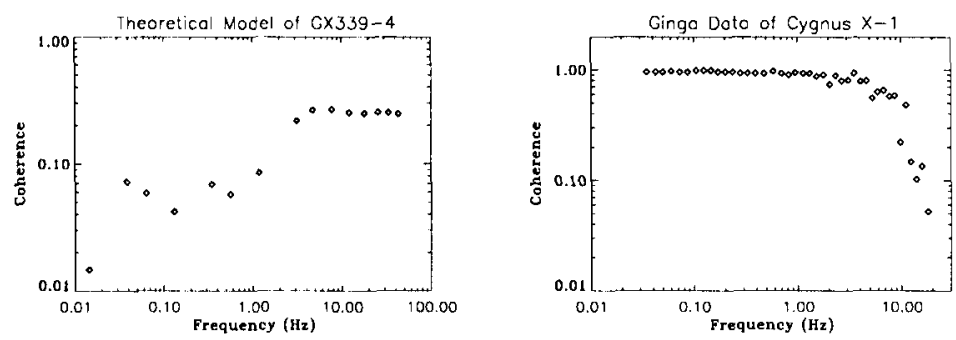

Figure 3. Left: Coherence of theoretical model with multiple flares (based on Nowak 1994). Right: Ginga data of Cyg X-1 (cf. Vaughan \& Nowak 1996) The Cyg X-1 loss of coherence is likely a noise artifact.

is not seen in Cyg X-1 (Fig. 3; Vaughan \& Nowak 1996), which shows near unity coherence between all well-observed energies. This also disagrees with the model of Manmoto et al. (1996). For that case the disturbance shocks upon reflection off of the disk inner edge, which is an inherently non-linear process, and thus leads to less than unity coherence for a stochastic source of variability.

As they currently stand, coronal models of Cyg X-1 have several problems. They predict too much soft X-ray flux, and they have yet to offer a viable explanation for the phase lags that also preserves unity coherence. ADD models also do not offer an explanation for the observed coherence, and they have yet to self-consistently calculate the observed "reflection" and line features.

Acknowledgments. We would like to acknowledge useful conversations with M. Begelman, P. Michelson, R. Staubert, C. Thompson, M. van der Klis, and all those who weren't allowed to ask questions after the talk, but were good enough to find me at coffee-break, especially F. Haardt and L. Maaraschi. M.A.N. gratefully acknowledges support from NASA grant NAG 5-3225.

\section{References}

Balbus, S., et al. 1996, this volume

Dove, J., Wilms, J., \& Begelman, M.C. 1996, ApJ, submitted

Haardt, F., \& Maraschi, L. 1993, ApJ, 413, 507

Hawley, J.C.., et al. 1996, this volume

Manmoto, T., et al. 1996, ApJ, 464, L135

Miller, M.C. 1995, ApJ, 441, 770

Miyamoto, S., et al. 1992 ApJ, 392, L21

Narayan, R., et al. 1996, this volume

Nowak, M.A. 1994, ApJ, 422, 688

Nowak, M.A. 1995, PASP, 718, 1207

Nowak, M.A., \& Vaughan, B.A., 1996 MNRAS, 280, 227

Vaughan, B.A., \& Nowak, M.A., 1996 ApJ, submitted

Zhang, S.N., et al. 1996, this volume 